\title{
Latinx Emerging Adults' Religious Identity, Ethnic Identity, and Psychological Well-Being
}

\author{
Isabella Nicole Schiro*, Carolyn McNamara Barry (D, Mary Jo Coiro and Emalee J. W. Quickel \\ Department of Psychology, Loyola University Maryland, Baltimore, MD 21210, USA; cbarry@loyola.edu (C.M.B.); \\ mcoiro@loyola.edu (M.J.C.); ejquickel@loyola.edu (E.J.W.Q.) \\ * Correspondence: inschiro@loyola.edu
}

Citation: Schiro, Isabella Nicole, Carolyn McNamara Barry, Mary Jo

Coiro, and Emalee J. W. Quickel. 2021. Latinx Emerging Adults' Religious Identity, Ethnic Identity, and Psychological Well-Being. Religions 12: 1073. https://doi.org/10.3390/ rel12121073

Academic Editor: Daniel H. Levine

Received: 31 August 2021

Accepted: 29 November 2021

Published: 3 December 2021

Publisher's Note: MDPI stays neutral with regard to jurisdictional claims in published maps and institutional affiliations.

Copyright: (c) 2021 by the authors. Licensee MDPI, Basel, Switzerland. This article is an open access article distributed under the terms and conditions of the Creative Commons Attribution (CC BY) license (https:// creativecommons.org/licenses/by/ $4.0 /)$.

\begin{abstract}
The current study examined associations among religious and ethnic identity exploration and commitment, and psychological well-being (PWB) among 683 Latinx emerging adults. A subset of data collected in the Multi-Site University Study of Identity and Culture was analyzed, focusing on three measures: (a) Multigroup Ethnic Identity Measure (MEIM), (b) Religious Identity Measure (adapted from MEIM), and (c) Scales of Psychological Well-Being-Short Form. Correlations indicated that PWB was positively related to religious and ethnic identity commitment, not exploration. Regression analyses indicated that commitment to religious or ethnic identity were positively associated with PWB, while exploration of religious or ethnic identity were not associated with PWB. In addition, religious identity exploration moderated the relation between ethnic identity exploration and commitment and PWB. These findings have implications for efforts to support the development of ethnic and religious identity among Latinx emerging adults.
\end{abstract}

Keywords: religious identity; ethnic identity; psychological well-being; emerging adulthood; Latinx

\section{Introduction}

The Latin $x^{1}$ population is one of the fastest growing ethnic groups in the United States with approximately 60.6 million individuals (Noe-Bustamante et al. 2020a) (see note 1). As members of a marginalized group, Latinx individuals face challenges, such as discrimination and acculturative stress, which threaten their overall psychological well-being (Cano et al. 2015). Latinx emerging adults (ages 18 to 29) are immersed in a tumultuous time marked by heightened identity development (Arnett 2015). High rates of mental health concerns (e.g., depression, anxiety) have been documented among emerging adults overall (American Psychiatric Association 2013), and Latinx emerging adults at even higher rates relative to other ethnic and age groups (Czeisler et al. 2020). A coherent sense of self and identity has been associated with higher levels of psychological wellbeing and functioning (Crocetti et al. 2014). While there are several identity domains (e.g., race, sexuality, gender) that are critical to emerging adults' developmental process, the current study focused on ethnic and religious identity, as these are especially salient in the Latinx culture (Gloria et al. 2004). For Latinx individuals, religiosity promotes mental health (Wilson et al. 2020), and ethnic identity development has been associated with psychological well-being (Smith and Silva 2011). Furthermore, ethnic identity is frequently linked to religiosity, especially in ethnic-minority samples (Vedder and Phinney 2014). Identity development typically involves engagement in varying levels of exploration, the act of exploring various identities, and commitment, the act of selecting and adhering to a specific identity (Marcia 1966). Therefore, the current study examined the associations between each form of identity (religious and ethnic) exploration and commitment and psychological well-being in Latinx emerging adults. 


\subsection{Religious Identity Development}

According to Abo-Zena and Ahmed (2014), religious identity development differs from religiosity, religious affiliation, and religious involvement because it reflects multiple, individualized, and sometimes contrasting pathways in the process of individuals forming their own perspectives on religious activities, practices, beliefs, and motivations. They further note that religious identity development is particularly salient during emerging adulthood due to increased autonomy and exploration. Emerging adults may begin to examine their religious beliefs and reconceptualize their religious values, beliefs, and identity.

Ethnic-minority youth are particularly likely to seek and value religious involvement to develop a sense of belonging and community (Abo-Zena and Barry 2013). Religious involvement, specifically church attendance, has been shown to increase feelings of belongingness for Latinx individuals (Jeong 2014) and may act as a foundation for the exploration and commitment needed for religious identity development. Research has indicated that religious practice and participation declines for Latinx emerging adults, but the importance of religious beliefs remains constant (Abo-Zena and Barry 2013). Research on associations between religiosity (i.e., religious affiliation, religious practices, and religious importance) and mental health among Latinx emerging adults is mixed, with some studies reporting no association (Lerman et al. 2018), others showing that lack of religiosity is associated with elevated depressive or anxiety symptoms in Latinx individuals (Lerman et al. 2018), or others suggesting religiosity plays a key role in promoting mental health (Da Silva et al. 2017). Therefore, the development and impact of religious identity development for Latinx emerging adults remains unclear.

\subsection{Ethnic Identity Development}

According to Phinney (1989), ethnic identity is a social construct that reflects an individual's experience of belonging or connection to one's ethnic group and the practice of cultural traditions. Phinney further notes that ethnic identity also refers to the way in which ethnic-minority group members perceive and navigate aspects of their ethnic background within the environment of a dominant culture. During emerging adulthood, individuals tend to experience greater independent exploration of ethnic identity (Neblett et al. 2019). While most emerging adults incorporate some degree of ethnic identity within their selfconcept, ethnic identity is generally more salient and significant for individuals from ethnic-minority groups (Phinney 1990). Ethnic-minority emerging adults encounter unique developmental demands and tasks due to societal devaluation of ethnic-minority groups and pressures to assimilate into the dominant society (Hughes et al. 2006). Therefore, Latinx ethnic identity development may be influenced by various external factors (e.g., ethnic socialization, intragroup marginalization, prejudice, discrimination), which may influence pathways of exploration and commitment in identity development. Overall, development of a positive ethnic identity has been identified as an essential component of positive adjustment and mental health in Latinx emerging adults (Umaña-Taylor et al. 2013).

\subsection{Identity Exploration, Formation, and Integration}

According to Erikson (1968), the psychosocial task of identity development involves resolving an identity crisis, and those who do not resolve this crisis would remain in a state of identity role confusion. In more recent decades with the delayed entrance into adult roles, Arnett (2015) contended that rather than adolescence, emerging adulthood (18-29) became the central time when young people engage in identity. In other words, there are more opportunities to explore different perspectives and reexamine beliefs.

Building upon the work of Erikson (1968), Marcia (1966) incorporated contextual factors such as family background, individual personality traits, and socialization practices as predictors of identity status. Thus, Marcia offered a broader model to understand identity development and formation by categorizing identity development into four identity statuses that align with distinct levels of exploration and commitment. However, it is important to note that the identity statuses proposed by Marcia's model of identity de- 
velopment have been criticized as overly static and failing to account for the nonlinear, continuous process of identity formation (Côte and Levine 1988; Stephen et al. 1992). Identity exploration is an individualized process that is influenced by numerous internal and external factors.

Identity formation is a central developmental task for emerging adults (Arnett 2015). Identity formation involves establishing autonomous values, beliefs, and personal characteristics in various identity domains. In addition, individuals must integrate identity domains that are central to their personality and beliefs in a way that is consistent across time and context. Identity integration can be defined as the degree to which social identities are perceived as compatible with one another (Cheng and Lee 2009; Huynh et al. 2011). Achieving identity integration has been linked to positive psychological well-being (Waterman 2007). Despite theoretical notions of the importance of identity integration across identity domains, little research has been conducted to examine multiple identity domains in the context of one another, especially in Latinx populations.

\subsection{Psychological Well-Being}

The instability that is characteristic of emerging adulthood has been linked with high rates of mental health concerns (e.g., depression, anxiety, substance use concerns; Schulenberg and Zarrett 2006). Identity formation is an especially important indicator of psychosocial well-being and adjustment (Erikson 1968). Identity commitment after supported exploration and acquisition of knowledge results in higher levels of overall psychological well-being, which fosters more stable mental health and stability (Luyckx et al. 2011).

The Latinx population experiences both external (e.g., discrimination, reception/ acceptance from society) and internal (e.g., bicultural stress) cultural stressors that affect their psychological and behavioral health (Grigsby et al. 2017). Cultural stressors have been associated with numerous adverse outcomes for Latinx peoples such as increased substance use (Castro et al. 2009), anxiety symptoms, and somatic complaints (Sirin et al. 2015). Most of the literature on Latinx psychological well-being has focused on negative psychological and behavioral outcomes but has not assessed the influence of specific factors on positive psychological outcomes, such as psychological well-being.

\subsection{Current Study}

The current study sought to examine the relations between Latinx emerging adults' religious identity, ethnic identity, and psychological well-being. We hypothesized that among a sample of Latinx emerging adults, exploration of, and commitment to, religious and ethnic identity would be independently associated with higher levels of psychological well-being. Given the insufficient literature on the joint role of ethnic and religious identity on psychological well-being, all potential interactions of ethnic and religious identity with psychological well-being were explored.

\section{Materials and Methods}

\subsection{Participants}

In the current study, participants were drawn from the Multi-Site University Study of Identity and Culture (MUSIC; Schwartz et al. 2009). A subsample of Latinx participants who were between 18 and 25 years of age was taken from the full sample based on specific inclusion and exclusion criteria. Because this study examined religious identity, individuals who reported "No Religion" or who identified as "Agnostic" or "Atheist" were excluded. The resultant sample of participants for the current study included 683 college-attending students (518 female, 165 male; $M_{\text {age }}=19.75, S D_{\text {age }}=1.73$ ), see Table $1 .^{2}$ 
Table 1. Participant Demographic Characteristics.

\begin{tabular}{lcc}
\hline Characteristic & $N$ & $\%$ \\
\hline Gender Identity & 165 & \\
Male & 518 & 75.8 \\
Female & & \\
\hline Religious Preference & 142 & 20.7 \\
$\quad$ Protestant & 480 & 70.3 \\
Roman Catholic & 4 & 0.6 \\
Jewish & 7 & 1.0 \\
Mormon & 3 & 0.4 \\
Jehovah Witness & 1 & 0.1 \\
Muslim & 2 & 0.3 \\
Hindu & 44 & 6.4 \\
Other & &
\end{tabular}

Note. Total $N=683$.

\subsection{Measures}

\subsubsection{Demographic Information}

Select demographics from the larger MUSIC dataset were used for the current study. Participants self-report their age, gender (male or female), ethnicity (seven major ethnicities and other), and religious preference (including 10 major religions, religious nonadherence (e.g., no religion, agnostic, atheist, and a write-in option).

\subsubsection{Multi-Group Ethnic Identity Measure}

Ethnic identity was assessed using the 12-item Multi-Group Ethnic Identity Measure (MEIM; Roberts et al. 1999), which examines (a) the extent to which one has considered the meaning of one's ethnicity (exploration; 5 items) and (b) the level of positive attachment to one's ethnic group (commitment; 7 items). The MEIM was adapted for MUSIC from being a 4-point to a 5-point Likert scale ranging from 1 (strongly disagree) to 5 (strongly agree). Two means scores were calculated, with higher scores indicating a higher level of exploration or commitment.

The MEIM has been shown to be internally consistent with alphas ranging from 0.81 (Goodstein and Ponterotto 1997) to 0.92 (Nguyen et al. 2015), signifying the scale's reliability across ethnic-minority groups of college-attending emerging adults (Yap et al. 2014). Overall ethnic identification scores have been shown to have some relation to ethnic self-concept (Phinney and Alpuria 1996), multicultural orientation or worldview (Ponterotto et al. 2003), and racial identity development (Goodstein and Ponterotto 1997). In general, MEIM scores have been associated positively with self-esteem, coping, sense of mastery, and optimism and associated negatively with loneliness and depression (Roberts et al. 1999). The majority of these correlations have been either small $(r<0.30)$ or medium ( $r=0.30$ to 0.49 ) effects. There are no current validity data to report for the exploration and commitment subscales. In the current study, Cronbach's alpha was 0.77 (MEIM-exploration) and 0.92 (MEIM-commitment).

\subsubsection{Religious Identity Measure}

Religious identity was assessed using the Religious Identity Measure, which was adapted from the MEIM and examines exploration ( 2 items) and commitment ( 7 items) to a specific religious group. Participants answered items on a 4-point Likert scale ranging from 1 (strongly disagree) to 4 (strongly agree). Sample items include, "I am active in organizations or social groups that include mostly members who are the same religions as me" (Exploration) and "I have a strong sense of belonging to my religion" (Commitment). Means scores were calculated, with higher scores indicating a higher level of exploration or commitment, respectively. Although no evidence of validity and reliability yet exists for this measure, such psychometric properties were established for its original measure focusing on ethnic identity (see previous paragraph). Furthermore, the items on the 
Religious Identity Measure are like items from other, similar, and well-validated measures such as the Multi-group Identity Measure-Religion (MIM-R; Ashdown et al. 2014). In the current study, Cronbach's alpha was 0.67 (exploration) and 0.94 (commitment).

\subsubsection{Psychological Well-Being}

Psychological well-being was assessed through the Scales of Psychological WellBeing, short form (SPWB; 18 items; Ryff and Keyes 1995) that reflects the six-domain model identified by Ryff (1989): self-acceptance, environmental mastery, positive relations, purpose in life, personal growth, and autonomy. This 7-point Likert scale was adapted for MUSIC to be a 6-point Likert scale ranging from 1 (strongly disagree) to 6 (strongly agree). After reverse coding eight items, a composite score is created by combining the results across the six subscales. Higher scores indicate higher levels of psychological well-being. In the current study, Cronbach's alpha was 0.81 .

\subsection{Procedure}

The Institutional Review Board (IRB) approved the original MUSIC (Schwartz et al. 2009) for data collection at 30 universities. The survey was advertised through emails and fliers from the psychology departments and other general education courses at each respective university by offering course credit (e.g., course requirement or extra credit); alternative ways to receive credit for students who did not consent to participating in the study. Informed consent was obtained from participants at each university site. Data were collected through an online link to the study website. All participants completed the surveys in the same order and were permitted to skip any items that they did not feel comfortable answering. All participants were appropriately debriefed and course credit was offered upon completion. No financial compensation was offered, but two universities offered a raffle of an iPod. Because IRB was obtained prior to data collection and the dataset was de-identified, a human subjects application was not required at Loyola University Maryland for secondary data analysis.

\section{Results}

The present study examined relations among religious identity exploration and commitment, ethnic identity exploration and commitment, and psychological well-being in Latinx emerging adults. Because this study examined religious identity, individuals who reported "No Religion" or who identified as "Agnostic" or "Atheist" were excluded. The resultant sample of participants for the current study included 683 college-attending Latinx students (518 female, 165 male; $M_{\mathrm{age}}=19.75, S D_{\mathrm{age}}=1.73$ ), with a majority identifying as Roman Catholic $(n=480)$ and Protestant $(n=126)$, see Table 1.

Table 2 provides descriptive statistics on main study variables (ethnic identity exploration, ethnic identity commitment, religious identity exploration, religious identity commitment, and psychological well-being), and Table 3 provides bivariate correlations among these variables.

As shown in Table 3, religious identity exploration was not correlated with psychological well-being, but as hypothesized, religious identity commitment was correlated significantly and positively with psychological well-being. Ethnic identity exploration was not correlated significantly with psychological well-being, but ethnic identity commitment was correlated significantly and positively with psychological well-being.

Next, a series of regression analyses were conducted to examine interactions between commitment and exploration of ethnic and religious identity. Using the PROCESS Macro for SPSS (Hayes and Rockwood 2017), these regression analyses included psychological well-being as the dependent variable, and religious exploration and commitment and ethnic identity exploration and commitment as independent variables and interaction terms. Due to the exploratory nature of the third hypothesis, six models were run to examine effects of each possible interaction of ethnic and religious identity exploration and commitment, as shown in Table 4 . The religious identity variables and the ethnic identity 
variables were mean-centered to reduce collinearity prior to creating the interaction terms. When significant moderation is found, PROCESS Macro provides the coefficient (as well as an indicator of whether the coefficient is statistically significant) for the association between the predictor (in this case ethnic identity) and the outcome (psychological well-being) at three levels of the moderator: more than 1 SD below the mean (termed "low"), within $1 S D$ above or below the mean (termed "moderate"), and more than $1 S D$ above the mean (termed "high").

Table 2. Descriptive Statistics of Study Variables.

\begin{tabular}{lcccc}
\hline Variable & $\boldsymbol{M}$ & SD & Skew & Kurtosis \\
\hline Ethnic Identity & & & & \\
$\quad$ Exploration & 3.31 & 0.9 & -0.17 & -0.48 \\
$\quad$ Commitment & 4.14 & 0.81 & -0.94 & 0.51 \\
\hline Religious Identity & & & & \\
$\quad$ Exploration & 2.4 & 0.82 & -0.001 & -0.59 \\
$\quad$ Commitment & 2.97 & 0.68 & -0.65 & 0.66 \\
\hline Psychological Well-Being & 80.74 & 11.39 & -0.48 & -0.3 \\
\hline
\end{tabular}

Note. Total $N=683$; Ethnic Identity scales range from 1 (Strongly Disagree) to 5 (Strongly Agree); Religious Identity scales range from 1 (Strongly Disagree) to 4 (Strongly Agree). Psychological Well-Being scales range from 1 (Strongly Disagree) to 6 (Strongly Agree).

Table 3. Bivariate Correlational Matrix of Study Variables.

\begin{tabular}{lccccc}
\hline Variables & $\mathbf{1}$ & $\mathbf{2}$ & $\mathbf{3}$ & $\mathbf{4}$ & $\mathbf{5}$ \\
\hline 1. Religious Identity Exploration & - & $0.56^{* *}$ & $0.30^{* *}$ & $0.21^{* *}$ & 0.02 \\
2. Religious Identity Commitment & & - & $0.13^{* *}$ & $0.24^{* *}$ & $0.28^{* *}$ \\
3. Ethnic Identity Exploration & & & - & $0.63^{* *}$ & 0.04 \\
4. Ethnic Identity Commitment & & & & - & $0.20^{* *}$ \\
5. Psychological Well-Being & & & & & - \\
\hline Note. ${ }^{* *} p<0.01$ (one-tailed); Listwise $N=602$. &
\end{tabular}

The first model examined the association between ethnic identity exploration and psychological well-being at different levels of religious identity exploration. As shown in Table 4, neither ethnic nor religious identity exploration was directly associated with psychological well-being. However, religious identity exploration moderated the association between ethnic identity exploration and psychological well-being. Specifically, there was a positive relation between ethnic identity exploration and psychological well-being, but only at high levels of religious identity exploration, see Figure 1.

The second model examined the association between ethnic identity commitment and psychological well-being at different levels of religious identity exploration. As shown in Table 3, ethnic identity commitment (not religious identity exploration) was directly associated with psychological well-being. Additionally, religious identity exploration moderated the association between ethnic identity commitment and psychological wellbeing. Specifically, there was a positive relation between ethnic identity commitment and psychological well-being at all three levels of religious identity exploration; however, the slope for those at low levels of religious exploration was less steep, see Figure 2.

The third and fourth models examined whether religious identity commitment moderated the association of psychological well-being with ethnic identity exploration (Model 3) and separately, ethnic identity commitment (Model 4). As shown in Table 4, although religious and ethnic identity commitment were associated with PWB, no significant interaction was found. 


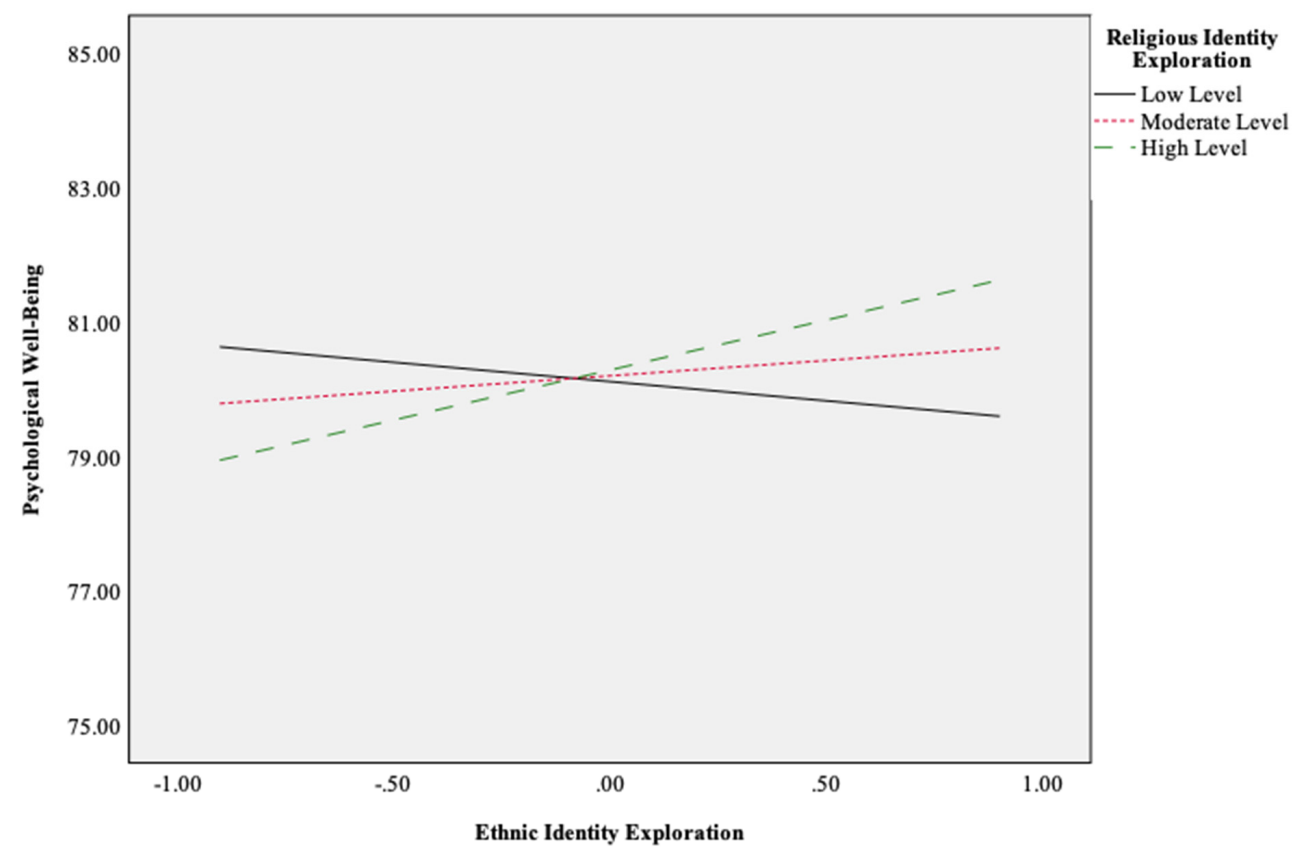

Figure 1. Model 1: Indirect Effect of Religious Identity Exploration on the Relation between Ethnic Identity Exploration and Psychological Well-Being.

Table 4. Hierarchical Multiple Regression Analyses Predicting Psychological Well-Being from Ethnic Identity Exploration or Commitment, Religious Identity Exploration or Commitment, and their Interaction.

\begin{tabular}{|c|c|c|}
\hline & \multicolumn{2}{|c|}{ Psychological Well-Being } \\
\hline & $b(\mathrm{SE})$ & $R^{2}$ \\
\hline Model 1 & & $0.03 * *$ \\
\hline Ethnic Identity Exploration (EIE) & $0.46(0.53)$ & \\
\hline Religious Identity Exploration (RIE) & $0.10(0.59)$ & \\
\hline $\mathrm{EIE} \times \mathrm{RIE}$ & $1.27(0.59) *$ & \\
\hline Model 2 & & $0.07^{* *}$ \\
\hline Ethnic Identity Commitment (EIC) & $2.89(0.57) * *$ & \\
\hline Religious Identity Exploration (RIE) & $-0.36(0.56)$ & \\
\hline $\mathrm{EIC} \times \mathrm{RIE}$ & $1.37(0.66) *$ & \\
\hline Model 3 & & $0.01 * *$ \\
\hline Ethnic Identity Exploration (EIE) & $0.08(0.49)$ & \\
\hline Religious Identity Commitment (RIC) & $4.72(0.66)^{* * *}$ & \\
\hline $\mathrm{EIE} \times \mathrm{RIC}$ & $0.59(0.69)$ & \\
\hline Model 4 & & $0.12 * *$ \\
\hline Ethnic Identity Commitment (EIC) & $2.08(0.57) * *$ & \\
\hline Religious Identity Commitment (RIC) & $4.10(0.67) * *$ & \\
\hline $\mathrm{EIC} \times \mathrm{RIC}$ & $0.39(0.75)$ & \\
\hline Model 5 & & $0.13^{* *}$ \\
\hline Religious Identity Exploration (RIE) & $-2.87(0.63)^{* *}$ & \\
\hline Religious Identity Commitment (RIC) & $6.71(0.78) * *$ & \\
\hline $\mathrm{RIE} \times \mathrm{RIC}$ & $1.86(0.66)^{* *}$ & \\
\hline Model 6 & & $0.10 * *$ \\
\hline Ethnic Identity Exploration (EIE) & $-2.52(0.61) * *$ & \\
\hline Ethnic Identity Commitment (EIC) & $5.70(0.75)^{* *}$ & \\
\hline $\mathrm{EIE} \times \mathrm{EIC}$ & $1.94(0.56)^{* *}$ & \\
\hline
\end{tabular}

Note. $b=$ unstandardized regression coefficient; $R^{2}=$ total variance explained in model. $N$ for Model $1=598 . N$ for Model $2=599 . N$ for Model $3=600 . N$ for Model $4=601 . N$ for Model $5=618 . N$ for Model $6=667 .{ }^{*} p<0.05$, ** $p<0.001$. 


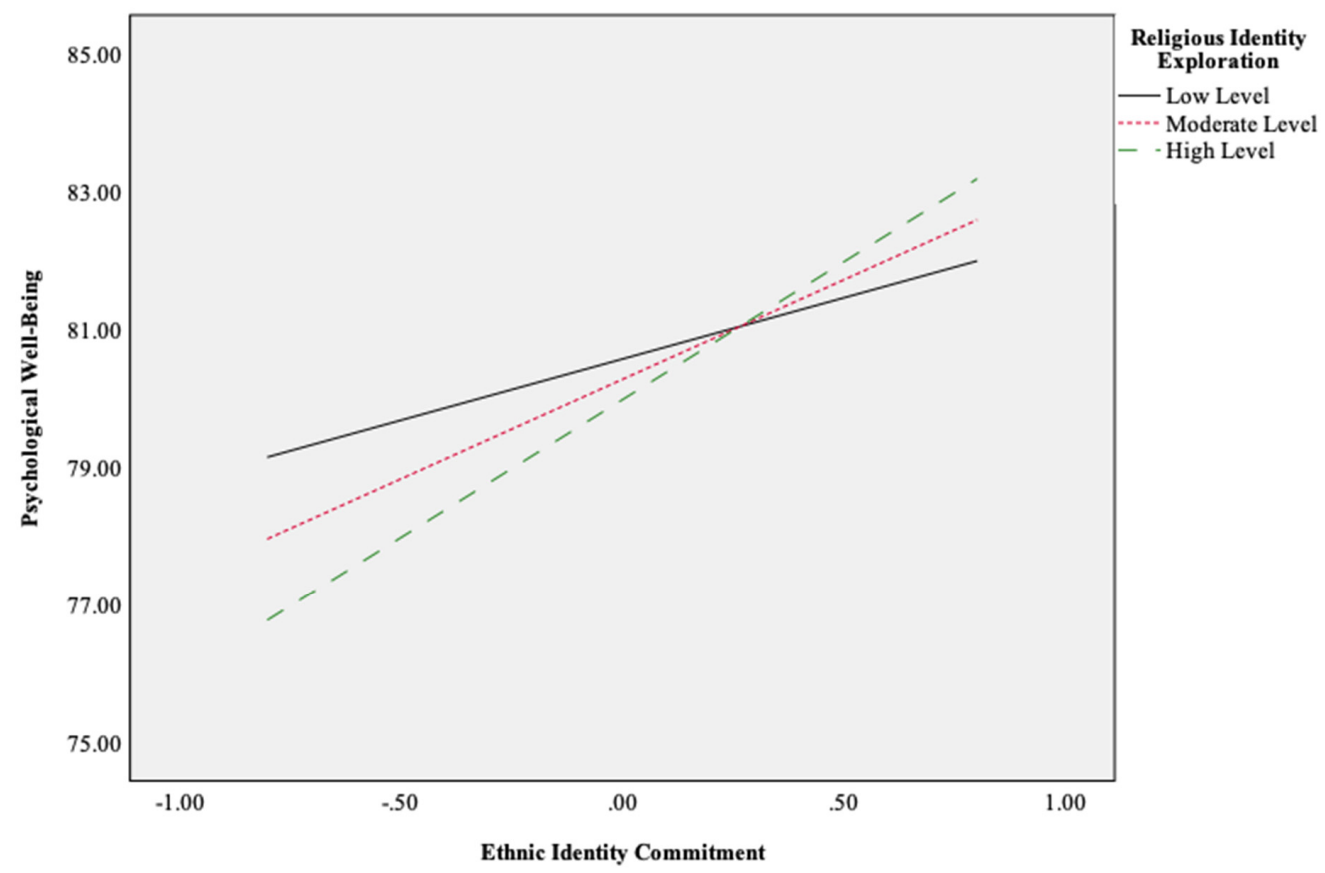

Figure 2. Model 2: Indirect Effect of Religious Identity Exploration on the Relation between Ethnic Identity Commitment and Psychological Well-Being.

The fifth model examined the association between religious identity commitment and psychological well-being at different levels of religious identity exploration. As shown in Table 4, religious identity commitment was significantly and positively associated with psychological well-being, but religious identity exploration was significantly and negatively associated with psychological well-being. Additionally, religious identity exploration moderated the association between religious identity commitment and psychological well-being. There was a positive relation between religious identity commitment and psychological well-being at all three levels of religious identity exploration, see Figure 3.

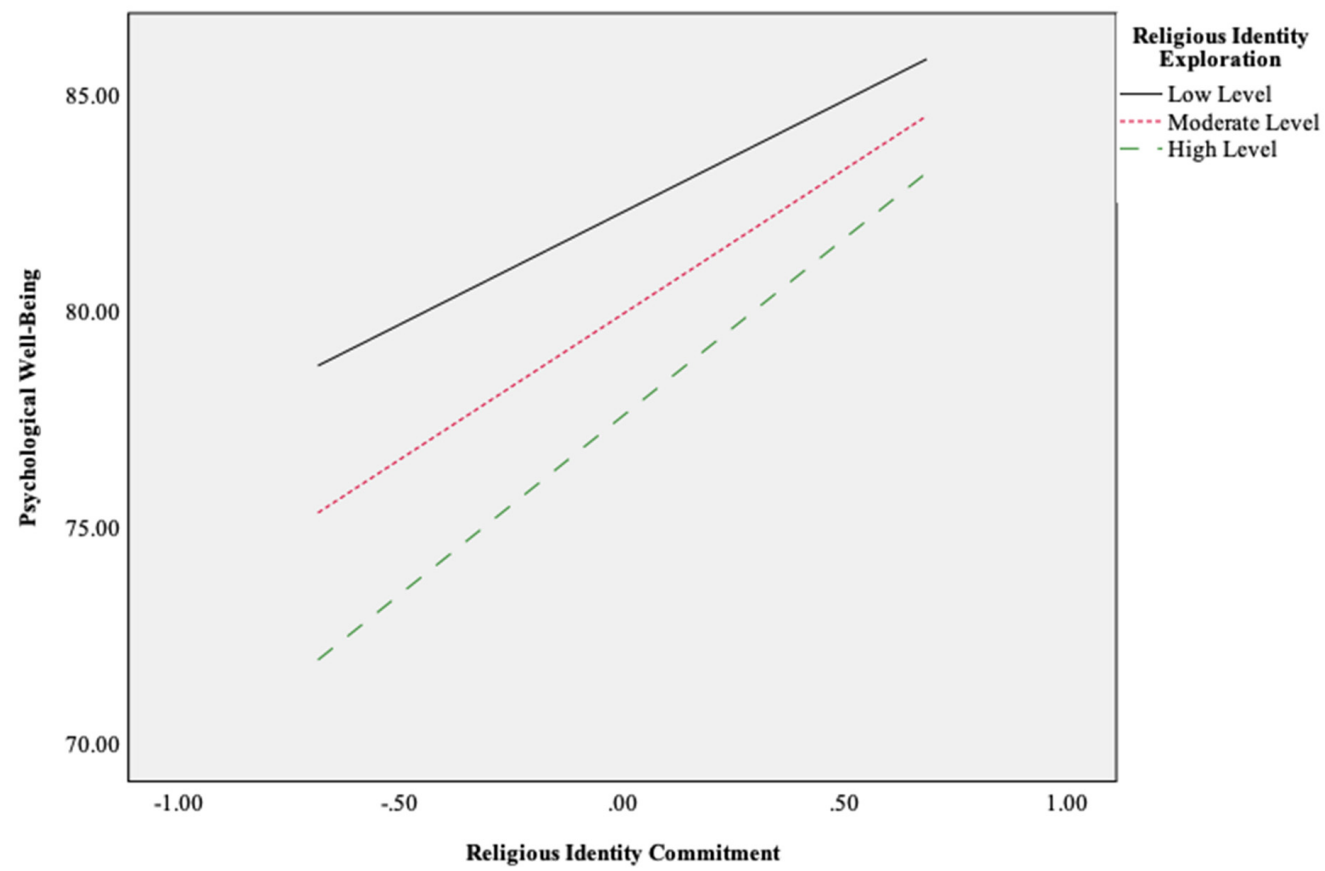

Figure 3. Model 5: Indirect Effect of Religious Identity Exploration on the Relation between Religious Identity Commitment and Psychological Well-Being. 
The sixth model examined the association between ethnic identity commitment and psychological well-being at different levels of ethnic identity exploration. As shown in Table 3, ethnic identity commitment was significantly and positively associated with psychological well-being, but ethnic identity exploration was significantly and negatively associated with psychological well-being. Additionally, ethnic identity exploration moderated the association between ethnic identity commitment and psychological well-being. There was a positive relation between ethnic identity commitment and psychological well-being at three levels of ethnic identity exploration, see Figure 4.

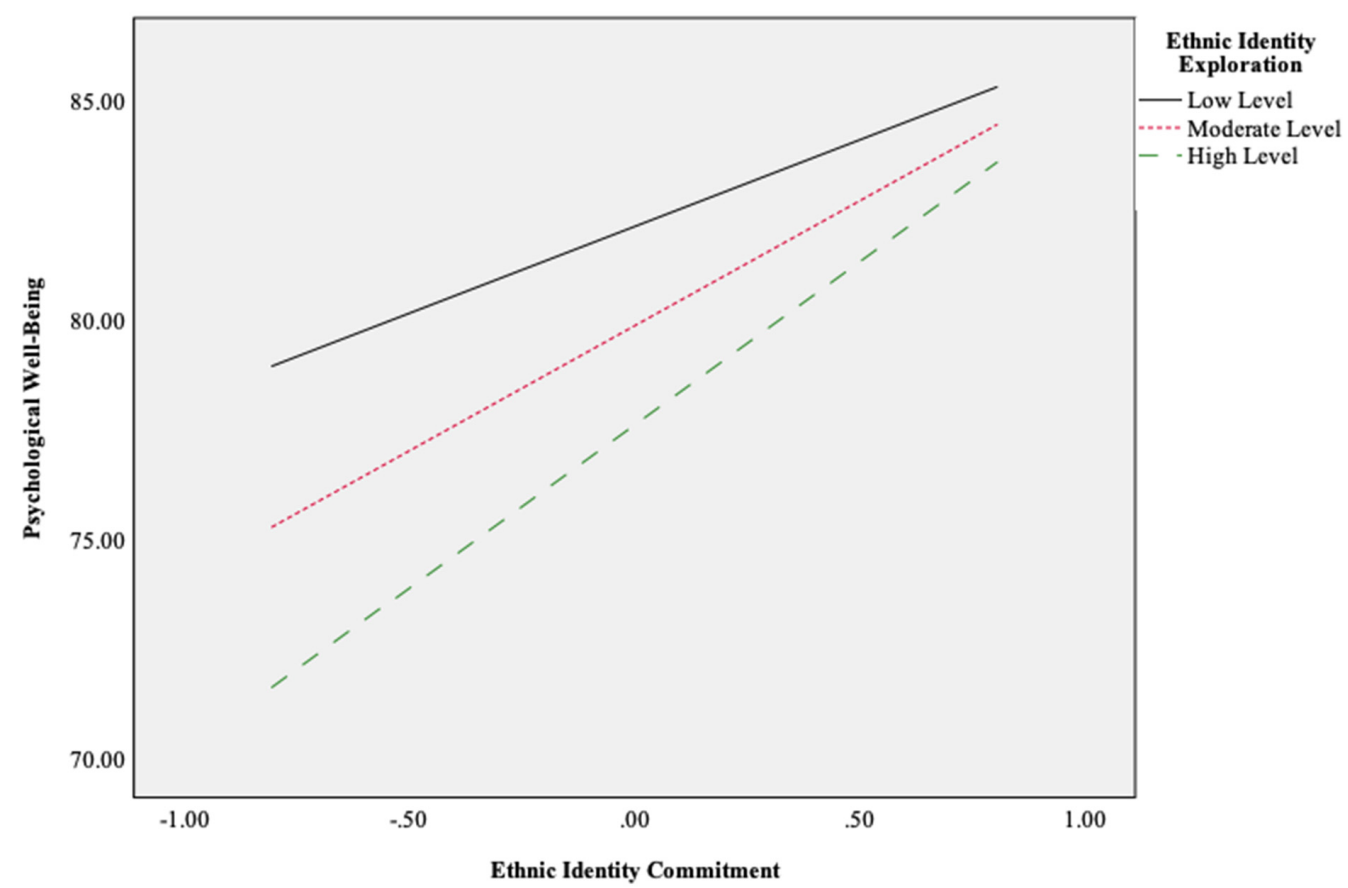

Figure 4. Model 6: Indirect Effect of Ethnic Identity Exploration on the Relation between Ethnic Identity Commitment and Psychological Well-Being.

\section{Discussion}

The purpose of the current study was to examine the associations among religious identity exploration and commitment, ethnic identity exploration and commitment, and psychological well-being in Latinx emerging adults.

\subsection{Psychological Well-Being, Religious Identity, and Ethnic Identity}

Contrary to the hypothesis, psychological well-being and identity exploration (both religious and ethnic) were not significantly related. It is possible that the Latinx population's overall level of exploration is lower than the general population, or that exploration is normative during emerging adulthood and thus is not associated with mental health. As hypothesized, psychological well-being and identity commitment (both religious and ethnic) were significantly and positively associated. These findings indicate that, in this sample of Latinx emerging adults, those who felt more committed in their religious or ethnic identity reported higher levels of psychological well-being than are those who were less committed in their identity. Previous research suggested that ethnic identity commitment may promote psychological well-being (Schwartz et al. 2009), and religiosity is helpful in promoting adjustment (specifically positive coping) for Latinx individuals (Da Silva et al. 2017). Therefore, our findings suggest that identity commitment provides structure and stability for emerging adults during a tumultuous period, which may support their psychological well-being.

It is also important to consider the potential pathways that led to the positive association between religious and ethnic identity commitment and psychological well-being. 
Because religious identity and ethnic identity are central cultural factors in the Latinx community (Gloria et al. 2004), it is possible that Latinx emerging adults may commit to these identities due to ethnic socialization (Hughes et al. 2006) or because they feel pressured to conform to the beliefs of their community (Neblett et al. 2019). On the other hand, social context factors, such as prejudice and discrimination, may also impact the salience and significance of committing to specific identities (Umaña-Taylor et al. 2014). Our findings agree with research suggesting that strength of identity commitment is significantly associated with psychological well-being (Vleioras and Bosma 2005), but differences in the process of exploration may not moderate the positive relation between commitment and psychological well-being. In sum, our findings suggest that psychological well-being is supported through identity commitment, regardless of the pathway of exploration.

Overall, these findings support prior literature suggesting that ethnic identity development for Latinx emerging adults and the strength of belonging to their ethnic group is associated with psychological well-being (Smith and Silva 2011). These findings also build upon the more limited literature on religiosity and adjustment among Latinx emerging adults (Da Silva et al. 2017) by offering a perspective of the role of religious identity on Latinx emerging adults' psychological well-being.

\subsection{Psychological Well-Being and Interactions of Religious and Ethnic Identity}

It is also important to review the interactions that emerged in the findings starting with those focused on identity domains (e.g., exploration) and then review interactions across identity domains. First, results from Models 5 and 6 suggested that identity exploration moderates the relation between identity commitment and psychological well-being for both religious identity and ethnic identity. In other words, unexpectedly, when the positive effects of identity commitment were controlled for, religious and ethnic identity exploration were significantly and negatively associated with psychological well-being. Therefore, our findings suggest that identity exploration may have a detrimental effect when experienced in isolation from identity commitment.

Results from Model 1 suggested a positive relation between ethnic identity exploration and psychological well-being, but only at high levels of religious identity exploration. Thus, Latinx emerging adults who engage in activities of religious searching at a high level may be more motivated to engage in activities of ethnic searching and experience heightened levels of psychological well-being. This finding may speak to the interwoven significance of these two identity domains in Latinx culture. As identity development progresses across adulthood (Arnett 2015), it is unclear if this positive relation between ethnic identity exploration and psychological well-being for those with high religious identity exploration would remain.

Results from Model 2 suggested a significant, positive relation between ethnic identity commitment and psychological well-being at all three levels of religious identity exploration; however, the slope for those at low levels of religious exploration was less steep. This finding suggests that there may be a process of identity integration occurring across identity domains, in that, these identities may be working and developing within the context of the other. While identity formation involves establishing autonomous values and beliefs across identity domains, identity integration involves the process of incorporating and managing multiple social identities (Syed and McLean 2016). It is possible that establishing commitment in one identity domain (in this case, ethnic identity commitment) allows for greater levels of identity integration with other identity domains (in this case, religious identity exploration). Identity commitment may provide the stability that promotes psychological well-being while exploring other identities. Overall, these results reaffirm the utility of an intersectional approach (Crenshaw 1989) to understanding identity factors and their impact on psychological well-being. 


\subsection{Limitations and Suggestions for Future Research}

The current study has limitations that should inform future research. First, this study utilized data from participants who identified as Latinx, but it did not account for the heterogeneity present within the Latinx population, such as national origin or level of acculturation. It is important to note that different backgrounds may emphasize ethnic identity and religious identity differently. Therefore, future research should continue to examine the Latinx population in a more nuanced manner and begin to parse apart how heterogeneity impacts aspects of identity development for specific subgroups in this community. Additionally, although the study's sample was large and drawn from multiple regions of the U.S., it was limited to college students; thus, these results may not apply to all Latinx emerging adults in the U.S. Further research should be conducted with emerging adults who are more vulnerable or who are living in non-college contexts that are structured and vocationally oriented to identify factors that promote healthy identity development and psychological well-being. Furthermore, our sample did not represent some demographic groups, including older emerging adults and those who identify as agnostic or atheist. Although the most common religious affiliations in the Latinx community and in this sample are Roman Catholic or Protestant (Pew Research Center 2007), it is possible that adults with other religious affiliations may explore and commit to religious identity differently.

Another limitation is that this study relied upon self-report data to assess emerging adults' psychological well-being, ethnic identity development, and religious identity development. Self-report data are convenient to obtain but also subjective, and participants' responses may be biased, overestimations, attempting to reduce cognitive dissonance, or pursuing social desirability (Bowman and Hill 2011). Future research should consider integrating qualitative data points in order to examine the lived experience of Latinx emerging adults more fully and not assume their experience based solely on numbers. Additionally, it is possible that these measures do not fully capture the nuances of Latinx experiences with religious identity development, ethnic identity development, and psychological well-being.

Given that the design of the current study was correlational and exploratory, we cannot examine causal or longitudinal relations among the variables. Further, the data were collected in 2008, and since that time, there have been dramatic shifts in U.S. immigration policies (Torres et al. 2018) and levels of discrimination (Lopez et al. 2018). For Latinx individuals, these policies exacerbate experiences of discrimination, perpetuate systemic concerns of access and opportunity, heighten fear and mistrust, and increase experiences of stress and trauma (Torres et al. 2018). Latinx individuals report that in recent years, due to increasing situations of discrimination, there are increasing instances of receiving unfair treatment because of their ethnic background, being called offensive names, being criticized for speaking Spanish in public, or being told to go back to their home country (Lopez et al. 2018). Therefore, these results may not generalize to Latinx emerging adults today. It is important to consider the findings from the current study, and future research, within the social context of the time.

Additionally, the measures used here may not capture the full range of ways that Latinx individuals pursue identity development or experience psychological well-being. Quantitative measures fail to capture the contextual and multifaceted aspects of identity formation. Quantitative measures fail to capture the story and influences behind the numbers. Future research should also integrate qualitative data (e.g., interviews, open response surveys) to examine the lived experience of Latinx emerging adults more fully, and not assume experience based solely on numbers. It will be important for future research to examine such factors individually and tease apart how identity development is related to nuanced factors of psychological well-being.

In conclusion, Latinx emerging adults constitute a specific subgroup within a highly visible ethnic minority group who frequently encounter discrimination and threats to their psychological well-being in the U.S. Understanding the associations between religious and ethnic identity and psychological well-being provides a more holistic view of protective factors for Latinx emerging adults. More work is required to research how psychological 
well-being can be promoted for emerging adults through the avenues of identity development, not only for the Latinx population but for other ethnic groups whose identity domains are often salient and interconnected. It is crucial for collaboration to occur between Latinx communities and researchers to find interventions, structural supports, and programs that promote psychological well-being, support mental health, and facilitate healthy identity development for Latinx emerging adults and the larger Latinx population.

Author Contributions: Conceptualization, I.N.S., C.M.B., M.J.C. and E.J.W.Q.; methodology, MUSIC collaborators (see Schwartz et al. 2009); formal analysis, I.N.S. and M.J.C.; writing—original draft preparation, I.N.S. and C.M.B.; writing—review and editing, I.N.S., C.M.B., M.J.C. and E.J.W.Q.; All authors have read and agreed to the published version of the manuscript.

Funding: This research received no external funding.

Institutional Review Board Statement: Ethical review and approval were waived for this study, due to conducting a secondary data analysis with deidentified data.

Informed Consent Statement: Informed consent was obtained from all subjects involved in the study.

Data Availability Statement: To gain access to the data used for this study, please contact the corresponding author, Isabella Schiro.

Acknowledgments: We are deeply grateful to Seth Schwartz and collaborators who created and organized the MUSIC project, as well as the university students throughout the U.S. who served as participants of this project.

Conflicts of Interest: The author declares no conflict of interest.

\section{Notes}

1 Throughout the paper we use the term Latinx. This pan-ethnic term describes the U.S. population of people tracing their roots to Latin America and Spain in an effort to be inclusive of all gender identities. However, there is evidence to show that this term is not the most preferred by those from this population (Noe-Bustamante et al. 2020b; Penaloza 2020; Pereira 2021). There is an ongoing dialogue within this population about language adaptations to practice inclusion.

2 PROCESS Macro, which was used for specific analyses, utilizes listwise analyses, which resulted in lower sample sizes in each model than in sample characteristics shown in Table 2.

\section{References}

Abo-Zena, Mona M., and Sameera Ahmed. 2014. Religion, spirituality, and emerging adults: Processing meaning through culture, context, and social position. In Emerging Adults' Religiousness and Spirituality: Meaning-Making in an Age of Transition. Edited by Carolyn McNamara Barry and Mona M. Abo-Zena. Oxford: Oxford University Press, pp. 220-36. [CrossRef]

Abo-Zena, Mona M., and Carolyn M. Barry. 2013. Religion and immigrant-origin youth: A resource and a challenge. Research in Human Development 10: 353-71. [CrossRef]

American Psychiatric Association. 2013. Diagnostic and Statistical Manual of Mental Disorders, 5th ed. Washington, DC: American Psychiatric Publishing.

Arnett, Jeffrey Jensen. 2015. Emerging Adulthood: The Winding Road from the Late Teens through the Twenties, 2nd ed. New York: Oxford University Press.

Ashdown, Brien K., Natalie Homa, and Carrie M. Brown. 2014. Measuring gender identity and religious identity with adapted versions of the Multigroup Ethnic Identity Measure-Revised. Journal of Educational and Developmental Psychology 4: 226-37. [CrossRef]

Bowman, Nicholas A., and Patrick L. Hill. 2011. Measuring how college affects students: Social desirability and other potential biases in college student self-reported gains. New Directions for Institutional Research 2011: 73-85. [CrossRef]

Cano, Miguel Ángel, Seth J. Schwartz, Linda G. Castillo, Andrea J. Romero, Shi Huang, Elma I. Lorenzo-Blanco, Jennifer B. Unger, Byron L. Zamboanga, Sabrina E. Des Rosiers, Lourdes Baezconde-Garbanati, and et al. 2015. Depressive symptoms and externalizing behaviors among Hispanic immigrant adolescents: Examining longitudinal effects of cultural stress. Journal of Adolescence 42: 31-39. [CrossRef] [PubMed]

Castro, Felipe González, Judith A. Stein, and Peter M. Bentler. 2009. Ethnic pride, traditional family values, and acculturation in early cigarette and alcohol use among Latino adolescents. The Journal of Primary Prevention 30: 265-92. [CrossRef]

Cheng, Chi-Ying, and Fiona Lee. 2009. Multiracial identity integration: Perceptions of conflict and distance among multiracial individuals. Journal of Social Issues 65: 51-68. [CrossRef] 
Côte, James E., and Charles Levine. 1988. A critical examination of the ego identity status paradigm. Developmental Review 8: 147-84. [CrossRef]

Crenshaw, Kimberlé. 1989. Demarginalizing the intersection of race and sex: A Black feminist critique of antidiscrimination doctrine, feminist theory and antiracist politics. University of Chicago Legal Forum 1989: 139-68.

Crocetti, Elisabetta, Wim H. J. Meeus, Rachel A. Ritchie, Alan Meca, and Seth J. Schwartz. 2014. Adolescent identity: Is this the key to unraveling associations between family relationships and problem behaviors? In Parenting and Teen Drug Use: The Most Recent Findings from Research, Prevention, and Treatment. Edited by Lawrence M. Scheier and William B. Hansen. New York: Oxford University Press, pp. 92-109.

Czeisler, Mark É., Rashon I. Lane, Emiko Petrosky, Joshua F. Wiley, Aleta Christensen, Rashid Njai, Matthew D. Weaver, Rebecca Robbins, Elise R. Facer-Childs, Laura K. Barger, and et al. 2020. Mental health, substance use, and suicidal ideation during the COVID-19 pandemic-United States, 24-30 June 2020. Morbidity and Mortality Weekly Report 69: 1049-57. [CrossRef] [PubMed]

Da Silva, Nicole, Frank R. Dillon, Toni Rose Verdejo, Mariana Sanchez, and Mario De La Rosa. 2017. Acculturative stress, psychological distress, and religious coping among Latina young adult immigrants. The Counseling Psychologist 45: 213-36. [CrossRef]

Erikson, Erik H. 1968. Identity, Youth, and Crisis. New York: W. W. Norton.

Gloria, Alberta M., Ester L. Ruiz, and Elisa M. Castillo. 2004. Counseling and psychotherapy with Latino and Latina clients. In Practicing Multiculturalism: Affirming Diversity in Counseling and Psychology. Edited by T. B. Smith. Boston: Allyn \& Bacon, pp. 167-89.

Goodstein, Renee, and Joseph G. Ponterotto. 1997. Racial and ethnic identity: Their relationship and their contribution to self-esteem. Journal of Black Psychology 23: 275-92. [CrossRef]

Grigsby, Timothy J., Myriam Forster, Alan Meca, Byron L. Zamboanga, Seth J. Schwartz, and Jennifer B. Unger. 2017. Cultural stressors, identity development, and substance use attitudes among Hispanic immigrant adolescents. Journal of Community Psychology 46: 117-32. [CrossRef]

Hayes, Andrew F., and Nicholas J. Rockwood. 2017. Regression-based statistical mediation and moderation analysis in clinical research: Observations, recommendations, and implementation. Behavior Research and Therapy 98: 39-57. [CrossRef] [PubMed]

Hughes, Diane, James Rodriguez, Emilie P. Smith, Deborah J. Johnson, Howard C. Stevenson, and Paul Spicer. 2006. Parents' ethnic-racial socialization practices: A review of research and directions for future study. Developmental Psychology 42: 747-70. [CrossRef]

Huynh, Que-Lam, Angela-MinhTu D. Nguyen, and Verónica Benet-Martínez. 2011. Bicultural identity integration. In Handbook of Identity Theory and Research. Edited by Seth J. Schwartz, Koen Luyckx and Vivian L. Vignoles. New York: Springer Science + Business Media, vols. 1-2, pp. 827-43.

Jeong, Hoi Ok. 2014. Religious involvement and group identification: The case of Hispanics in the United States. The Social Science Journal 51: 21-29. [CrossRef]

Lerman, Shir, Molly Jung, Elva M. Arredondo, Janice M. Barnhart, Jianwen Cai, Sheila F. Castañeda, Martha L. Daviglus, Rebeca A Espinoza, Aida L. Giachello, Kristine M. Molina, and et al. 2018. Religiosity prevalence and its association with depression and anxiety symptoms among Hispanic/Latino adults. PLoS ONE 13: e0185661. [CrossRef]

Lopez, Mark Hugo, Ana Gonzalez-Barrera, and Jens Manuel Krogstad. 2018. More Latinos Have Serious Concerns about Their Place in America under Trump. Pew Research Center. Available online: https://www.pewresearch.org/hispanic/2018/10/25/morelatinos-have-serious-concerns-about-their-place-in-america-under-trump/ (accessed on 4 November 2021).

Luyckx, Koen, Hans De Witte, and Luc Goossens. 2011. Perceived instability in emerging adulthood: The protective role of identity capital. Journal of Applied Developmental Psychology 32: 137-45. [CrossRef]

Marcia, James E. 1966. Development and validation of ego-identity status. Journal of Personality and Social Psychology 3: 551-58. [CrossRef] [PubMed]

Neblett, Enrique W., Jr., Wendy D. Roth, and Moin Syed. 2019. Ethnic and racial identity development from an interdisciplinary perspective: Introduction to the special issue. Emerging Adulthood 7: 79-84. [CrossRef]

Noe-Bustamante, Luis, Mark Hugo Lopez, and Jens Manuel Krogstad. 2020a. U.S. Hispanic Population Surpassed 60 Million in 2019, But Growth Has Slowed. Pew Research Center, July 7. Available online: https://www.pewresearch.org/fact-tank/2020/07/07/u-shispanic-population-surpassed-60-million-in-2019-but-growth-has-slowed/ (accessed on 4 November 2021).

Noe-Bustamante, Luis, Lauren Mora, and Mark Hugo Lopez. 2020b. About One-in-Four U.S. Hispanics Have Heard of Latinx, But Just 3\% Use It. Pew Research Center, August 11. Available online: https:/ /www.pewresearch.org/hispanic/2020/08/11/about-one-infour-u-s-hispanics-have-heard-of-latinx-but-just-3-use-it/ (accessed on 4 November 2021).

Nguyen, Chi P., Y. Joel Wong, Linda P. Juang, and Irene J. K. Park. 2015. Pathways among Asian Americans' family ethnic socialization, ethnic identity, and psychological well-being: A multigroup mediation model. Asian American Journal of Psychology 6: 273-80. [CrossRef]

Penaloza, Marisa. 2020. Latinx Is a Term Many Still Can't Embrace. NPR, October 1. Available online: https://www.npr.org/2020/10/01 /916441659/latinx-is-a-term-many-still-cant-embrace (accessed on 4 November 2021).

Pereira, Evan Odegard. 2021. For Most Latinos, Latinx Does Not Mark the Spot. New York Times, June 15. Available online: https:/ / www.nytimes.com/2021/06/15/learning/for-most-latinos-latinx-does-not-mark-the-spot.html (accessed on 4 November 2021). 
Pew Research Center. 2007. Changing Faiths: Latinos and the Transformation of American Religion. April 25. Available online: http:/ / www.pewhispanic.org/2007/04/25/changing-faiths-latinos-and-the-transformation-of-american-religion/ (accessed on 11 January 2019).

Phinney, Jean S. 1989. Stages of ethnic identity development in minority group adolescents. Journal of Early Adolescence 9: 34-49. [CrossRef]

Phinney, Jean S. 1990. Ethnic identity in adolescents and adults: Review of research. Psychological Bulletin 108: 499-514. [CrossRef]

Phinney, Jean S., and Linda L. Alpuria. 1996. At the interface of cultures: Multiethnic/multiracial high school and college students. The Journal of Social Psychology 136: 139-58. [CrossRef]

Ponterotto, Joseph G., Denise Gretchen, Shawn O. Utsey, Thomas Stracuzzi, and Robert Saya Jr. 2003. The Multigroup Ethnic Identity Measure (MEIM): Psychometric review and further validity testing. Educational and Psychological Measurement 63: 502-15. [CrossRef]

Roberts, Robert E., Jean S. Phinney, Louise C. Masse, Y. Richard Chen, Catherine R. Roberts, and Andrea Romero. 1999. The structure of ethnic identity of young adolescents from diverse ethnocultural groups. The Journal of Early Adolescence 19: 301-22. [CrossRef]

Ryff, Carol D. 1989. Happiness is everything, or is it? Explorations on the meaning of psychological well-being. Journal of Personality and Social Psychology 57: 1069-81. [CrossRef]

Ryff, Carol D., and Corey Lee M. Keyes. 1995. The structure of psychological well-being revisited. Journal of Personality and Social Psychology 69: 719-27. [CrossRef] [PubMed]

Schulenberg, John E., and Nicole R. Zarrett. 2006. Mental health during emerging adulthood: Continuity and discontinuity in courses, causes, and functions. In Emerging Adults in America: Coming of Age in the 21st Century. Edited by Jeffrey J. Arnett and Jennifer L. Tanner. Washington, DC: American Psychological Association, pp. 135-72. [CrossRef]

Schwartz, Seth J., Byron L. Zamboanga, Robert S. Weisskirch, and Liliana Rodriguez. 2009. The relationships of personal and ethnic identity exploration to indices of adaptive and maladaptive psychosocial functioning. International Journal of Behavioral Development 33: 131-44. [CrossRef]

Sirin, Selcuk R., Lauren Rogers-Sirin, Jessica Cressen, Taveeshi Gupta, Sammy F. Ahmed, and Alfredo D. Novoa. 2015. Discriminationrelated stress effects on the development of internalizing symptoms among Latino adolescents. Child Development 86: 709-25. [CrossRef]

Smith, Timothy B., and Lynda Silva. 2011. Ethnic identity and personal well-being of people of color: A meta-analysis. Journal of Counseling Psychology 58: 42-60. [CrossRef]

Stephen, Joanne, Eugene Fraser, and James E. Marcia. 1992. Moratorium-achievement (MAMA) cycles in lifespan identity development: Value orientations and reasoning system correlates. Journal of Adolescence 15: 283-300. [CrossRef]

Syed, Moin, and Kate C. McLean. 2016. Understanding identity integration: Theoretical, methodological, and applied issues. Journal of Adolescenc 47: 109-18. [CrossRef]

Torres, Stephanie A., Catherine DeCarlo Santiago, Katherine Kaufka Walts, and Maryse H. Richards. 2018. Immigration policy, practices, and procedures: The impact on the mental health of Mexican and Central American youth and families. American Psychologist 73: 843. [CrossRef] [PubMed]

Umaña-Taylor, Adriana J., Megan O’Donnell, George P. Knight, Mark W. Roosa, Cady Berkel, and Rajni Nair. 2014. Mexican-origin early adolescents' ethnic socialization, ethnic identity, and psychosocial functioning. The Counseling Psychologist 42: 170-200. [CrossRef] [PubMed]

Umaña-Taylor, Adriana J., Katharine H. Zeiders, and Kimberly A. Updegraff. 2013. Family ethnic socialization and ethnic identity: A family-driven, youth-driven, or reciprocal process? Journal of Family Psychology 27: 137-46. [CrossRef]

Vedder, Paul, and Jean S. Phinney. 2014. Identity formation in bicultural youth: A developmental perspective. In The Oxford Handbook of Multicultural Identity. Edited by Verónica Benet-Martínez and Ying-Yi Hong. New York: Oxford University Press, pp. 335-54.

Vleioras, Georgios, and Harke A. Bosma. 2005. Are identity styles important for psychological well-being? Journal of Adolescence 28: 397-409. [CrossRef] [PubMed]

Waterman, Alan S. 2007. Doing well: The relationship of identity status to three conceptions of well-being. Identity: An International Journal of Theory and Research 7: 289-307. [CrossRef]

Wilson, Elizabeth J., Kymberley K. Bennett, Jillian M. R. Clark, Kadie M. Harry, Denisse Tiznado, Kalon R. Eways, Anahi Ramirez, and Ricardo M. Marte. 2020. The interaction between fatalism and religious attendance is negatively associated with mental health-related quality of life in Hispanic/Latino Americans low in acculturation. North American Journal of Psychology 22: 521-42.

Yap, Stevie C. Y., M. Brent Donnellan, Seth J. Schwartz, Su Yeong Kim, Linda G. Castillo, Byron L. Zamboanga, Robert S. Weisskirch, Richard M. Lee, Irene J. K. Park, Susan Krauss Whitbourne, and et al. 2014. Investigating the structure and measurement invariance of the Multigroup Ethnic Identity Measure in a multiethnic sample of college students. Journal of Counseling Psychology 61: 437-46. [CrossRef] [PubMed] 